\title{
Absence of Evidence of Transmission of Coronavirus Disease 2019 from a Young Child to Mother Despite Prolonged Contact
}

\author{
H. Nassih ${ }^{1}$ - K. El Fakiri ${ }^{1}$ - I. Ait Sab ${ }^{1}$
}

Received: 20 May 2020 / Accepted: 3 June 2020 / Published online: 13 June 2020

(C) Dr. K C Chaudhuri Foundation 2020

To the Editor: Many reports speculate that children -being most of the time asymptomatic- are the driving force behind the spread of the coronavirus disease 2019 (COVID-19). This paper aims to add more proof against children vectoring the SARS-CoV-2 virus.

A well-appearing two-years-old girl presented to our hospital for SARS-CoV-2 testing. Her father (himself infected by a colleague) tested positive for COVID-19 a day before. Their last encounter was a week ago, after which the father was out of town. She did not contact anyone else than her mother for the last two weeks. The mother and daughter were asymptomatic with unremarkable physical examination. Nasal swab for SARS-CoV-2 RT-PCR was positive for the child and negative for the mother. Twenty-four hours later, the mother underwent a second RT-PCR combined with IgM/IgG antibody rapid test. Both tests were negative for SARS-CoV-2. The mother was confirmed disease-free. The father's condition worsened, and he got admitted to the hospital; Also there was no other confirmed COVID-19 family member to take care of the child, so she had to stay under the care of her uninfected mother. Airborne and contact precautions were mandatory. Daily checkups, as well as weekly SARS-CoV-2 RT-PCR and serology controls of both mother and child, were carried out for one month. Both kept in good health. The girl's RTPCR became negative by the tenth day of diagnosis, while SARS-CoV-2 RT-PCR and serology controls of the mother stayed negative up to twenty-eight days of follow-up.

Recent reports are contradicting the previous understanding that the transmission of COVID-19 among children is one

H. Nassih

houda.ped@gmail.com

1 Department of Pediatrics, Child and Mother Hospital, Mohammed VI University Hospital Center, Marrakesh Medical and Pharmacy Faculty, Caddy Ayad University, Marrakesh, Morocco of the several factors driving the pandemic [1]. In fact, in the majority of family clusters, it is the parents who infect their children and not the other way [2,3]. Usually, younger children take part in fewer daily social activities than teenagers, and their contact opportunities with carriers or patients should be lower, resulting in a lower risk of getting the COVID-19 illness [4]. We describe a single case in a child with her mother not proven to have the infection despite the long close contact. Based on a single case, it is difficult to conclude that children do not act as transmitters of COVID-19. This report suggests that children may not be as infectious of SARS-CoV-2 as adults, though in reality, it is still not known.

\section{Compliance with Ethical Standards}

Consent The patient's parents' consent to publication has been obtained.

Conflict of Interest None.

\section{References}

1. Hu Z, Song C, Xu C, et al. Clinical characteristics of 24 asymptomatic infections with COVID-19 screened among close contacts in Nanjing. China Sci China Life Sci. 2020;63:706-11.

2. Qian G, Yang N, Ma AHY, et al. COVID-19 transmission within a family cluster by presymptomatic carriers in China. Clin Infect Dis [Internet]. 2020 Mar 23. Available at: https://www.ncbi.nlm.nih.gov/ pmc/articles/PMC7184331/. Accessed May 19, 2020.

3. Jeng M-J. Coronavirus disease 2019 in children: Current status. J Chin Med Assoc [Internet]. 2020 May 4. Available at: https:// www.ncbi.nlm.nih.gov/pmc/articles/PMC7199766/. Accessed May 19, 2020.

4. Kelvin AA, Halperin S. COVID-19 in children: The link in the transmission chain. Lancet Infect Dis [Internet]. 2020 Mar 25. Available at: https://www.ncbi.nlm.nih.gov/pmc/articles/PMC7156154/. Accessed May 19, 2020.

Publisher's Note Springer Nature remains neutral with regard to jurisdictional claims in published maps and institutional affiliations. 